\title{
Low multiplicity technique for GRB observation by LHAASO-WCDA
}

\author{
H.R. Wu ${ }^{1}$, H.H.He ${ }^{1}$ for the LHAASO collaboration \\ ${ }^{1}$ Institute of High Energy Physics, Chinese Academy of Sciences, Beijing, 100049, China \\ E-mail: hhh@ihep.ac.cn
}

\begin{abstract}
Detection of $\mathrm{GeV}$ photons from GRBs is crucial in understanding the most violent phenomenon in our universe. Due to the limited effective area of space-born experiment, very few GRBs are detected with $\mathrm{GeV}$ photons. Large area EAS experiments at high altitude can reach a much larger effective area around $10 \mathrm{GeV}$, for which single particle technique is usually used to lower the threshold energy but its sensitivity is poor due to losing primary direction information. To reach an energy threshold as low as $10 \mathrm{GeV}$ and keep the primary direction information at the same time, low multiplicity trigger is required, but random coincidences rather than cosmic ray showers overwhelms the signals, and it is a great challenge for traditional trigger logic and reconstruction algorithm to discriminate the signals from the noises. A new method is developed for LHAASOWCDA(Large High Altitude Air Shower Observatory-Water Cherenkov Detector Array) to work under low multiplicity mode. With this technique, the LHAASO detector can even work under multiplicity as low as 2 while keeping the direction information at the same time. The sensitivity and expectation of LHAASO-WCDA with low multiplicity technique to GRBs are presented.
\end{abstract}

The 34th International Cosmic Ray Conference,

30 July- 6 August, 2015

The Hague, The Netherlands

\footnotetext{
*speaker
} 


\section{Introduction}

Gamma Ray Bursts(GRBs) are among the most powerful events in the Universe, and have been the subject of observational studies from radio to multi-GeV energies. Satellites with instruments sensitive to hard gamma-rays, such as CGRO and Fermi LAT, have extended the observations from $30 \mathrm{MeV}$ to tens of GeV. GRB130427A [1] that was observed up to $94 \mathrm{GeV}$, or $126 \mathrm{GeV}$ once corrected for redshift, shows that GRBs are capable of producing very-high-energy photons. On the present, several GRBs have been observed above $10 \mathrm{GeV}$ [2] [3] [4] [5] [6]. It is unknown up to what energy the spectrum extends, as present-day observations are limited by effective area, in the case of space-based instruments, and by slewing constraints and energy threshold for groundbased Imaging Air Cherenkov Telescopes. Studying the spectrum beyond $10 \mathrm{GeV}$ is crucial in understanding GRB mechanisms themselves, and also allows us to probe cosmological phenomena such as extra-galactic background light (EBL) and it may be used to constrain Lorents invariance violation.

Currently three major classes of high-energy detectors exist: Satellite detectors, Imaging Atmospheric Cherenkov Telescopes (IACTs) and Extensive Air Shower (EAS) particle detector arrays. Satellites can observe very wide fields of view (e.g. 2.4sr or $19 \%$ of $4 \pi$ sr for Fermi LAT) and have close to a $100 \%$ operational duty cycle. On the other hand, the limited physical size of satellites prevents them from obtaining enough statistics to reach energies greater than tens of $\mathrm{GeV}$. Operating above $50 \mathrm{GeV}$ IACTs that have been designed for fast slewing $(1 \mathrm{~min})$. EAS detector arrays, such as WCDA, benefit from a very large field of view ( $2 \mathrm{sr}$ or $16 \%$ of $4 \pi \mathrm{sr}$ ) and near $100 \%$ duty cycle that will allow for observations in the prompt phase. They are also sensitive to energies beyond those covered by satellites. EAS observatories, in particular WCDA, are thus useful high-energy GRB detectors that complement the observations by satellites such as Fermi.

For EAS detectors, at present, two methods can be used to analysis the sensitivity and capabilities of detection of GRBs by WCDA: Shower mode method, Single particle technique (SPT) and Low multiplicity technique. Shower model method is a regular analysis method, threshold-energy is about $100 \mathrm{GeV}$, but GRBs with $100 \mathrm{GeV}$ photons are very few. SPT can detect GRBs with $10 \mathrm{GeV}$ photon but poor in sensitivity due to losing direction information. So taking advantage of characteristic of trigger mode of WCDA, a new method, low multiplicity technique is developed for GRBs detection, which can reach such energy like tens of $\mathrm{GeV}$ and reserve direction information at the same time. In this proceeding we will present the sensitivity and capabilities of low multiplicity technique for detection of GRBs by WCDA and show the observatory's ability to measure possible high-energy emission from GRBs.

\section{WCDA experiment and trigger mode}

Targeting gamma astronomy in energy band from $100 \mathrm{GeV}$ to $30 \mathrm{TeV}$, the WCDA is one of the major components of the LHAASO, covering an area 90,000 $\mathrm{m}^{2}$, has been proposed to be built at Daocheng County (4300 m a.s.1.), GanZizhou, SiChuan, China. Is is made of four ponds, $150 \times 150 \mathrm{~m}^{2}$ each. Each pond is divided into 900 cells $\left(5 \times 5 \mathrm{~m}^{2}\right.$ each partitioned by $0.5 \mathrm{~mm}$-thick black curtains made of black polyethylene lines, with a effective water depth of $4 \mathrm{~m}$ ) seen by one 
PMT located at the cell floor center and looking up to detect the direct Cherenkov light produced by the relativistic particles of the showers.

The measured counting rate was about $36 \mathrm{kHz}$ for each cell, which is much higher than the noise of PMT itself. So a new trigger technique based on "Triggerless" is proposed for large area WCDA: each PMT will output a L1 (Level 1) single trigger signal of $250 \mathrm{~ns}$ after hit and over threshold, and if there is another over-threshold signal in the same $250 \mathrm{~ns}$ period, which will be taken as a new signal and trigger signal will extend $250 \mathrm{~ns}$; namely the total array is divided into 81 trigger cluster $(60 \mathrm{~m} \times 60 \mathrm{~m}$ each, including $12 \times 12=144$ PMTs $)$ Horizontally and vertically, and then judge whether hit multiplicity is bigger than 12 at the falling edge of the clock of each trigger cluster. When any trigger cluster satisfies this selection, then output one L2 trigger signal and produce total trigger. In this trigger system, if single counting rate produced by cosmic ray is less than $50 \mathrm{kHz}$, random coincidence trigger rate produced by single counting can be controlled smaller than $1 \mathrm{kHz}$, and trigger rate of $70 \mathrm{kHz}$ produced by air shower $(>10 \mathrm{GeV})$ is expected.

\section{Low multiplicity technique}

\subsection{Challenge in low multiplicity trigger for WCDA}

In order to lower the threshold energy and reserve the primary direction information at the same time for GRBs detection, Low multiplicity technique is produced taking advantage of LHAASOWCDA trigger technique based on "triggerless", wide field of view and full duty cycle, only observatory array like LHAASO-WCDA with these three characteristics can do this. We have known the counting rate was $36 \mathrm{kHz}$ for each PMT, and about $10^{8} \mathrm{~Hz}$ for the whole array $(3600$ cells), which is much higher than signal from cosmic ray, so it's impossible for WCDA to trigger or reconstruct correctly the true events. Then how to discriminate the signals from the noise, it's a great challenge for traditional trigger logic and reconstruction algorithm? A new method is developed for LHAASO-WCDA to work under low multiplicity mode.

Lowering down the huge background rate from single rate is crucial for this method. Three steps are take to realize this goal. firstly, we take the GRB alert as an "event" trigger for follow-up observation. As we know, typical delay of a GRB alert is about 1 minute, when a GRB alert comes, DAQ takes the GRB alert as an "event" trigger for low multiplicity technique and stores all the data in the pipeline and data of a certain time duration after the alert before reconstructing the shower core and direction for follow up observation. This implies that the GRB position is known from other observations. The time and duration of the burst are also assumed known, which allows one to efficiently reject the background by defining a restrictive time window. There is no any problem and hardware-free for WCDA. Secondly, we localize the shower, namely lower the total single rate. we can consider events with a distance from center of the array $<50 \mathrm{~m}$, and with zenith angle $<40^{\circ}$, after doing so, the total number of noise hits is reduced by a factor of 10 , but it's still too high for a multiplicity as low as 3 . At last, we shrink the trigger time window through hit time transformation. After this transformation, the coincidence time window can be reduced by a factor of $\sim 30$, in which the average number of noise hits can be reduced by a factor of $\sim 30$, from 3 to 0.1 , it's good for low multiplicity trigger.

In this method, all hits are to be saved, and the running time window should be applied on each hit, otherwise, shower hits can be separated into adjacent windows. 


\begin{tabular}{|c|c|c|c|}
\hline & nhit $\geq 1\left(m^{2}\right)$ & nhit $\geq 2\left(m^{2}\right)$ & nhit $\geq 3\left(m^{2}\right)$ \\
\hline $10 \mathrm{GeV}$ & 36986 & 4699 & 1710 \\
\hline $20 \mathrm{GeV}$ & 94533 & 18941 & 8214 \\
\hline $50 \mathrm{GeV}$ & 297410 & 88885 & 46175 \\
\hline $100 \mathrm{GeV}$ & 537151 & 224280 & 139644 \\
\hline
\end{tabular}

Table 1: effective area at different energy for low multiplicity

\subsection{Signal simulation for low multiplicity technique}

Gamma-ray showers are simulated with CORSIKA with an $E^{-2.0}$ spectrum at different energy. The detector response model developed for WCDA is used at an altitude of $4300 \mathrm{~m}$ using a GEANT4 based code. The lowest energy for primary gamma showers was set to $10 \mathrm{GeV}$. The signal rate in the low multiplicity technique is the number of PMT hit after detector response and before direction and position reconstruction.

The signal rate $\mathrm{S}$ is given by:

$$
S(\theta)=\int d E \frac{d N}{d E} A_{\text {eff }}^{\text {lowmultiplicity }}(E, \theta)
$$

Where $d N / d E$ is the photon spectrum and $A_{\text {eff }}^{\text {lowmultiplicity }}$ is the detector effective area. Depends on several variables and here only energy $E$ and zenith angle are treated. In this proceeding, only results of $\theta=0$ are presented, and other direction will be considered later. The effective area of WCDA for gamma rays for different low multiplicity is shown in the Table1.

\subsection{Background for low multiplicity technique}

Galactic cosmic rays are simulated with CORSIKA for multiple species with an $E^{-2.62}$ spectrum: protons, $\mathrm{He}, \mathrm{C}, \mathrm{O}, \mathrm{Ne}, \mathrm{Mg}, \mathrm{Si}, \mathrm{Fe}$. The galactic cosmic ray spectrum is reweighted to measurement by J.R. Hoerandel [8]. Background rate mainly includes occasional noise from cosmic rays and random coincidence noise. Fig 1 is the distribution of hit number from cosmic ray background by simulation, from this figure, we can infer the occasional noise rate is $4.3 \times 10^{6} \mathrm{~Hz}$ for $n$ hit $\geq 1,1.6 \times 10^{6} \mathrm{~Hz}$ for $n$ hit $\geq 2$ and $8.4 \times 10^{5} \mathrm{~Hz}$ for $n$ hit $\geq 3$ by fitting the distribution of number of hits with a power law.

For random coincidence noise, firstly, we take the pipe line with a time duration of $200 \mathrm{sec}-$ onds, to save all the data when a GRB alert comes and after the alert. Secondly, we localize the shower, considering events with a distance from the center of $<50 \mathrm{~m}$, then the total Number of noise hits is reduced by a factor of 10 , i.e. from 30 hits $\left(10^{8} \mathrm{~Hz} \times 300 \times 10^{-9} \mathrm{~s}=30\right.$ hits $)$ reduced to 3 hits in trigger time window of $300 \mathrm{~ns}$. The random coincidence noise rate is about $8 \mathrm{e} 7 \mathrm{~Hz}$ for nhits $\geq 3$, about 300 times higher than shower rate (with direction information). Secondly, we shrink the trigger time window, namely perform hit time transformation: GRB direction cosines are $(1, \mathrm{~m}, \mathrm{n})$, for each hit $(\mathrm{x}, \mathrm{y}, \mathrm{z}, \mathrm{t})$, we can define $t r=t-(l x+m y+n z) / C$, where $\mathrm{C}$ is light velocity in vacuum, Automatically removed those far from GRB direction and shower direction information is obtained without direction reconstruction. After this transformation, the coincidence time window can be reduced from $300 \mathrm{~ns}$ to $10 \mathrm{~ns}$, in which the average number of noise hits is reduced from 3 to 0.1 , good for low multiplicity trigger. For low multiplicity trigger, the running time window was 


\begin{tabular}{|l|c|c|c|}
\hline & occasional noise rate $(\mathrm{Hz})$ & random coincidence noise rate $(\mathrm{Hz})$ & background rate $(\mathrm{Hz})$ \\
\hline nhit $\geq 1$ & $4.3 \times 10^{6}$ & $10^{8}$ & $1.04 \times 10^{8}$ \\
\hline nhit $\geq 2$ & $1.6 \times 10^{6}$ & $9.5 \times 10^{6}$ & $1.11 \times 10^{7}$ \\
\hline nhit $\geq 3$ & $8.4 \times 10^{5}$ & $4.6 \times 10^{5}$ & $1.30 \times 10^{6}$ \\
\hline
\end{tabular}

Table 2: Background rate at different low multiplicity

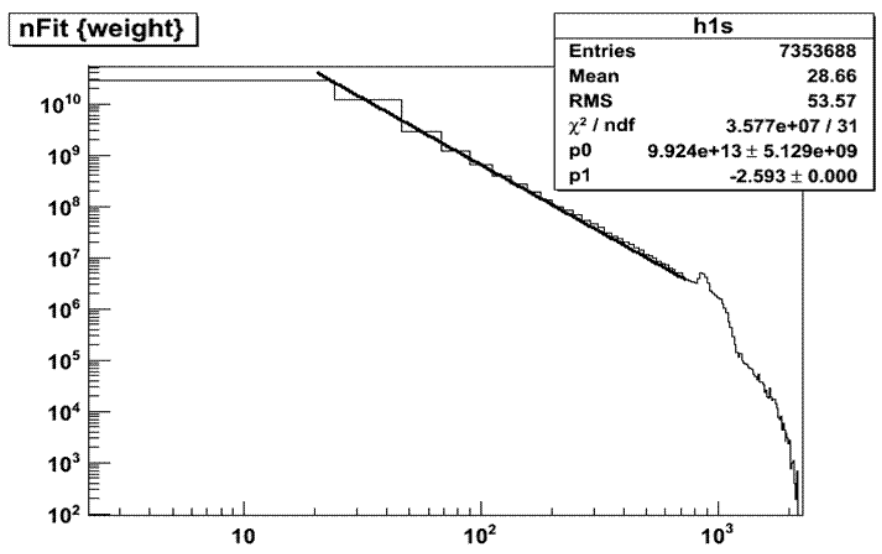

Figure 1: Distribution of number of hit. Solid line is a power law fit to the number of hits to infer low multiplicity due to threshold effect.

applied on each hit, then the random coincidence rate is about $5 \mathrm{e} 5 \mathrm{~Hz}$ (nhits $\geq 3$ for mean noise hit of 0.1), lowering down a factor of 100. For different low multiplicities, the random coincidence noise rate and the Background rate are presented in the following Table2.

\subsection{Sensitivity of low multiplicity technique to GRBs}

For low multiplicity, the sensitivity of WCDA to GRBs depends on a number of factors, including the GRB emission time scale, emission spectrum and redshift, as well as on the signal and background estimation of the experiment. To calculate WCDA's sensitivity, we simulate gamma ray spectrum according to the power-law $d N / d E \propto E^{-2}$ with an arbitrary reference flux normalization. This injection spectrum can be weighted for any other spectral shapes. In which we take into account attenuation of VHE gamma rays due to interaction with extragalactic background light, the Franceschini et al. [7] model is used.

Given a signal rate $S(\theta)$, background rate B then the significance of a given observation is :

$$
\operatorname{Sig}=S(\theta) T_{90} / \sqrt{B T_{90}}=\sqrt{T_{90} / B} \int d E \frac{d N}{d E} A_{\text {eff }}^{\text {lowmultiplicity }}(E, \theta)
$$

We have used various spectra of the type $d N / d E \propto E^{-\gamma}$ with sharp high-energy cutoffs to determine the sensitivity of the low multiplicity technique to GRBs. The sensitivity is defined as the flux detectable at $5 \sigma$ significance. A range of spectral indices gamma between -3 and -1 and a range of cutoffs between $10 \mathrm{GeV}$ and $10 \mathrm{TeV}$ were tested, and effects of the EBL are also considered.

Figure 2 shows WCDA's sensitivity curve of different GRB emission spectra on the expected sensitivity of WCDA using the low multiplicity (nhit $\geq 3$ ) calculated with equation compared to 


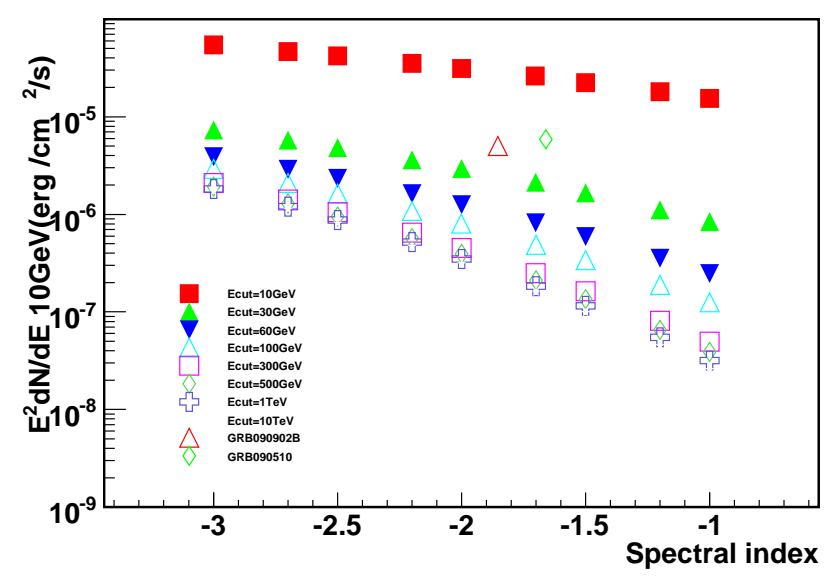

Figure 2: Sensitivity using the low multiplicity as a function of spectral index. The $5 \sigma$ discovery potential is shown as a function of spectral index for various values of a sharp high-energy spectral cutoff. The duration of the burst is fixed to $1 \mathrm{~s}$ and the zenith angle is fixed to 0 . Data from 2 GRBs are corrected for duration and inserted for comparison [3] [4]

GRBs that have been detected by Fermi LAT. Assuming that the burst occurs at a zenith angle of $0^{\circ}$ and lasts 1 second at a distance of redshift of $z=0.5$ [7]. Data for GRBs 090510 and 090902b, extracted from [3] and [4] are shown for comparison. We conclude that the most promising cases for detection with high significance are GRBs such as GRB 090510 and GRB 090902b if the highenergy cutoff is above $30 \mathrm{GeV}$. Fermi LAT observations of these two GRBs were made up to 30 $\mathrm{GeV}$ without any indication of a cutoff. If high-energy emission from GRBs extends beyond 30 $\mathrm{GeV}$, then WCDA will become even more significant due to limited physical size of Fermi LAT.

\section{Scientific prospect and conclusions}

A new method was developed to detect GRBs at energy as low as $10 \mathrm{GeV}$ and reserve source direction information using EAS array, like LHAASO-WCDA. From above analysis, we can conclude, WCDA, will have the capability of detecting GRBs at high energies. The simulations presented in this proceeding show that WCDA will be able to detect GRBs with characteristics similar to those of some of the brightest GRBs seen by Fermi LAT. As opposed to Fermi LAT, with a fixed physical size, the effective of the method of the low multiplicity increases with energy. Thus this method will expand upon the energy sensitivity of current detectors. Also WCDA is a wide field of view detector with near $100 \%$ duty cycle, it will be able to make GRB observation in the prompt phase. WCDA, in union with satellite or other ground based detectors, will be able to measure the high-energy GRB components including a possible high-energy cutoff. Important astrophysical information will be deduced from spectral cutoffs such as the Lorentz boost factor of GRB jets, the effects of the EBL and the maximum energy to which GRBs accelerate particles.

For low multiplicity technique, how to pick up the true events and analysis the data? This is still the question to be solved. In this proceeding, we present the result with GRBs alert information, at further step, we also can do without GRBs alert and work alone taking advantage of large field of view of WCDA, then CPU power maybe is a huge challenge. 


\section{Acknowledgments}

This work is supported in China by NSFC (NO.11375210, No.11375224, No.11405181, No.11475190), the Chinese Academy of Science, Institute of High Energy Physics, the Key Laboratory of Particle Astrophysics, CAS and in Italy by the istituto nazionale di fisica nucleare (INFN).

\section{References}

[1] S.Zhu et al., GCN Circ. 14471(2013)

[2] A.A. Abdo et al., Science 323(2009) 1688-1693

[3] M. Ackermann et al., ApJ. 716(2010) 1178-1190

[4] Abdo A.A. et al., ApJ. 706 (2009) L138-L144

[5] M. Ackermann et al., ApJ. 729(2011) 114

[6] M. Ackermann et al., ApJ. Suppl (2013)

[7] A. Franceschini, G. Rodighiero, and M. Vaccari, A\&A 487,837-852(2008)

[8] J.R. Hoerandel, astro-ph/0407118 